\title{
Approximation Algorithm for Multi-input and Multi-output System using Generalized Block Pulse Integration Operational Matrices
}

\author{
Joon-Hoon Park \\ Dept. of Control \& Instrumentation Eng., Korea National University of \\ Transportation, Korea \\ jhpark@ut.ac.kr
}

\begin{abstract}
In the control theory, a system has one or more input and output signals. With the development of control theory, a modern control system is carried out in the state space, and can process multi-input and multi-output (MIMO) systems. According to the complexity of a control system, a control system may have been described by a high order differential equation or transfer function, thus control system analysis and design becomes more difficult. Therefore a study to obtain a low order model with the dynamic characteristics similar to a high order system is very meaningful. In this paper, an approximation algorithm for multivariable system based on the generalized block pulse transform is presented. To solve the functional differential equation and transfer function, the generalized block pulse function is used. The algorithm adopted in this paper has shown that computational results are more accurate and convenient rather than conventional block pulse transform.
\end{abstract}

Keywords: multivariable system, high order model, approximation, generalized block pulse transform, integration operational matrices

\section{Introduction}

Today, control systems are widely used in various fields which are not limited to engineering and industry. For example, modern cars use a combination of electronics and mechanism to develop self-driving car system. A home heating system is a simple control system consisting of a thermostat containing expansion. With the development of digital computer technology, the digital computer has become an important part of the control systems. The digital computers in control systems are used for applications such as industrial machine system control, common household appliances and spacecraft system design. By the way, all of these control systems may have been expressed by a differential equation or transfer function. The transfer function model is derived from the Laplace transform. And the definition of transfer function is extended to a multivariable system that has multiple inputs and outputs. The block diagram representation of a MIMO system with $n$ inputs and $m$ outputs are shown in Figure 1. Unlike a single input and single output system, a MIMO system can be represented as matrix polynomial form which is more complex to handle. In a MIMO system, a differential equation may be used to describe the relationship between a pair of input and output variables, when all other inputs are set to zero. In 1969, Harmuth introduced the block pulse function. The block pulse function is a set of orthogonal functions with piecewise constant values. But it has not received any attention with regard to practical applications. Afterwards with Walsh and Haar function, the block pulse function has been studied and applied extensively as a fundamental set of functions for a system control and communication field. Later, the generalized block pulse function was derived to replace the repeated applications of the conventional block pulse function [1]. The generalized block pulse operational matrix can better approximate 
integral operators in time domain analysis. In this paper, a MIMO high order system is transformed into its corresponding algebraic expression and is replaced with a lower order model using generalized block pulse operational matrix and transform. The results of the proposed algorithm are more accurate and convenient rather than the conventional block pulse transform.

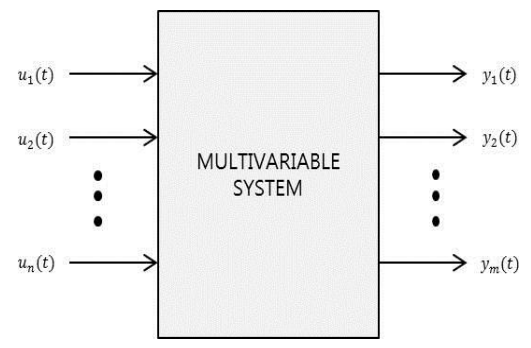

Figure 1. Block Diagram of A MIMO System

\section{Conventional and Generalized Block Pulse Function}

\subsection{Conventional Block Pulse Function}

The applications of orthogonal pulse functions fir the analysis of system science was first introduced by Corrington in 1973. Corrington constructed Walsh tables to solve differential equatioin, then Walsh operational matrix has been applied to various problems in control theory and engineering. The complete set of block pulse function over the time interval $[0, T)$ can be defined as [2]

$$
\varphi_{i}(t)=\left\{\begin{array}{cc}
1, & \frac{(i-1)}{m} T \leq t \leq \frac{i}{m} T, i=1,2, \ldots, m \\
0, & \text { otherwise }
\end{array}\right.
$$

Figure 2 shows a set of block pulse functions with $m=4$.

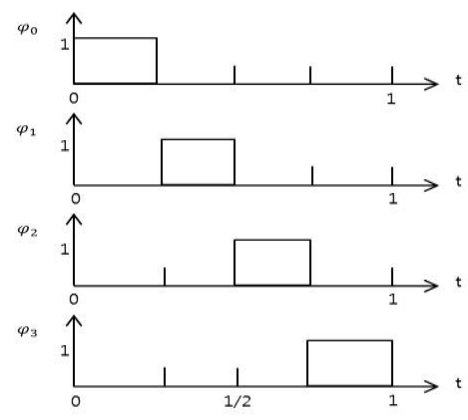

Figure 2. Block Pulse Functions with $M=4$

The block pulse function is orthogonal with each other in the interval $t \in[0, T)$

$$
\int_{0}^{T} \varphi_{i}(t) \varphi_{i}(t) d t=\left\{\begin{array}{l}
h, \text { for } i=j \\
0, \text { for } i \neq j
\end{array}\right.
$$

where $i, j=1,2, \ldots, m$

For any piecewise continuous function $f(t)$, it can be expanded into a block pulse function series in the sense of minimizing the mean square error between $f(t)$ and its approximation.

$$
f(t) \cong \hat{f}(t)=\sum_{i=0}^{m-1} F_{i} \varphi_{i}(t)
$$


where $0 \leq t<T$ and $F_{i}$ is the block pulse coefficient with respect to the ith block pulse function $\varphi_{i}(t)$.

$$
F_{i}=\frac{m}{T} \int_{0}^{T} f(t) \varphi_{i}(t) d t
$$

And the original function $f(t)$ can be approximated by a piecewise constant function $\hat{f}(t)$. The criterion of this approximation is the mean square error between $f(t)$ and $\hat{f}(t)$ in interval $t \in[0, T)$.

$$
\varepsilon=\frac{1}{T} \int_{0}^{T}\left(f(t)-\sum_{j=1}^{m} F_{j} \varphi_{j}\right)^{2} d t
$$

For example, we can expand $f(t)=t^{2} / 2$ into its block pulse series in the interval $t \in[0,1)$ with $m=8$. The results are shown in Figure 3 .

$$
\begin{aligned}
& F_{0}=\frac{1}{2}\left[f(0)+f\left(\frac{1}{8}\right)\right]=\frac{1}{256} \\
& F_{1}=\frac{1}{2}\left[f\left(\frac{1}{8}\right)+f\left(\frac{2}{8}\right)\right]=\frac{5}{256} \\
& F_{2}=\frac{1}{2}\left[f\left(\frac{2}{8}\right)+f\left(\frac{3}{8}\right)\right]=\frac{13}{256} \\
& F_{3}=\frac{1}{2}\left[f\left(\frac{3}{8}\right)+f\left(\frac{4}{8}\right)\right]=\frac{25}{256} \\
& F_{4}=\frac{1}{2}\left[f\left(\frac{4}{8}\right)+f\left(\frac{5}{8}\right)\right]=\frac{41}{256} \\
& F_{5}=\frac{1}{2}\left[f\left(\frac{5}{8}\right)+f\left(\frac{6}{8}\right)\right]=\frac{61}{256} \\
& F_{6}=\frac{1}{2}\left[f\left(\frac{6}{8}\right)+f\left(\frac{7}{8}\right)\right]=\frac{85}{256} \\
& F_{7}=\frac{1}{2}\left[f\left(\frac{7}{8}\right)+f\left(\frac{8}{8}\right)\right]=\frac{113}{256}
\end{aligned}
$$

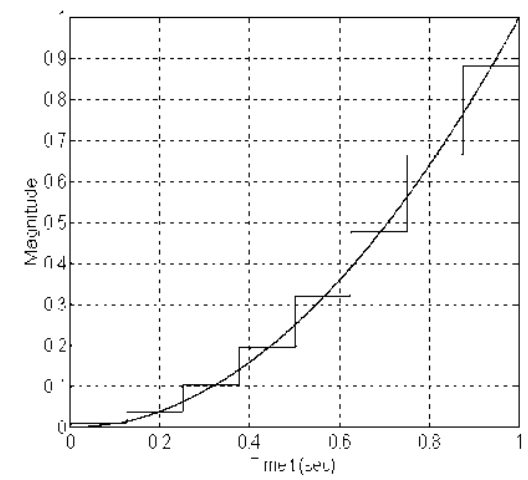

Figure 3. Block Pulse Series Expansion of $F(T)=T^{2} / 2$ With $M=8$

In order to expand the integral of a function into its block pulse series, we first have to consider the integral of each single block pulse function $\varphi_{i}(t)$ [3]. For the case of $t \in[0$, $i$ h), we have

$$
\int_{0}^{t} \varphi_{i}(t) d t=0
$$

For the case of $t \in[i \mathrm{~h},(i+1) \mathrm{h})$, we have

$$
\int_{0}^{t} \varphi_{i}(t) d t=\int_{0}^{i h} \varphi_{i}(t) d t+\int_{i h}^{t} \varphi_{i}(t) d t=t-i h
$$


And in the case of $t \in\left[(i+1) \mathrm{h}, t_{f}\right)$, we have

$$
\int_{0}^{t} \varphi_{i}(t) d t=\int_{0}^{i h} \varphi_{i}(t) d t+\int_{i h}^{(i+1) h} \varphi_{i}(t) d t+\int_{(i+1) h}^{t} \varphi_{i}(t) d t=h
$$

From the above discussion, the block pulse series for the integral of all the $m$ block pulse functions can be written in a compact form [4].

$$
\int_{0}^{t} \varphi_{i}(t) d t \cong P \varphi_{i}(t)
$$

where the operational matrix $P$ :

$$
P=\frac{h}{2}\left[\begin{array}{ccccc}
1 & 2 & 2 & \cdots & 2 \\
0 & 1 & 2 & \cdots & 2 \\
0 & 0 & 1 & \cdots & 2 \\
\vdots & \vdots & \vdots & \ddots \\
0 & 0 & 0 & \cdots & 1
\end{array}\right]
$$

The $P$ matrix is defined by the integral operation of the block pulse functions. The matrix has a regular form of an upper triangular matrix. The $\mathrm{k}$ th row is obtained by shifting the first row to the right. And all $m$ eigenvalues of the upper triangular matrix can also be seen as $h / 2$. On the basis of the integration operational matrix, the integral of a function $f(t)$ can be easily expressed using the following block pulse series [5].

$$
\int_{0}^{t} f(t) d t \cong \int_{0}^{t} F^{T} \varphi_{i}(t) d t \cong F^{T} P \varphi_{i}(t)
$$

And we can get multiple integral of the block pulse function as [6]

$$
\begin{array}{r}
\int_{0}^{t} \ldots \int_{0}^{t} f(t) d t^{k} \ldots d t \\
=\int_{0}^{t} \ldots\left(\int_{0}^{t}\left(\int_{0}^{t} f(t) d t\right) d t\right) \ldots d t \\
\cong F^{T} P^{k} \varphi_{i}(t)
\end{array}
$$

Figure 4 shows the integral of block pulse functions with $m=4$.

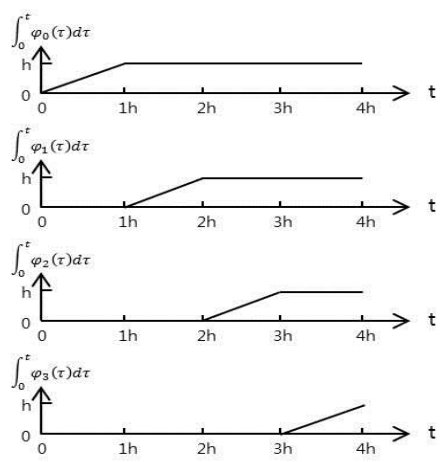

Figure 4. Integral of Block Pulse Functions With $M=4$

\subsection{Generalized Block Pulse Function and Operational Matrix}

The integration operational matrix of block pulse series is important for a control system analysis and applications. In order to improve the accuracy of block pulse coefficients obtained from the integral operation rule, we can consider some better 
approximation of an equation (2.19). The improvement and modification of the integration operational matrix of block pulse series can be expressed as $\bar{P}$

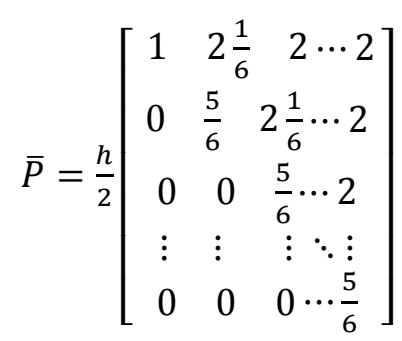

It is easy to express the integral of a function $f(t)$ into its block pulse series using this improved integration operational matrix $\bar{P}[6]$.

$$
\int_{0}^{t} f(t) d t \cong \int_{0}^{t} F^{T} \varphi_{i}(t) d t \cong F^{T} \bar{P} \varphi_{i}(t)
$$

During the process of solving the control system, multiple integrals appear frequently and the results obtained by the integration operational matrix $P$ and $\bar{P}$ have still some calculative defects. The $k$ time's integral of a single block pulse function $\varphi_{i}(t)$ can be written as

$$
\int_{0}^{t} \ldots \int_{0}^{t} \varphi_{i}(t) d t \ldots d t=\frac{1}{(k-1) !} \int_{0}^{t}(t-\tau)^{k-1} \varphi_{i}(\tau) d \tau
$$

Since $P^{k}$ or $\bar{P}^{k}$ are powers of integration operational matrix, the calculation will increase greatly when the dimension of matrices become larger and errors or calculative defects will be accumulated at each computational step. This idea was realized as generalized integration operational matrix of $\bar{P}_{g}$ in the equation (2.24). To solve these problems, the idea to transform multiple integrals into algebraic operations in one step was introduced by Wang in 1982 [7]. It was shown that the integration operational matrix of conventional block pulse series is a special case for the generalized block pulse operational matrix with $k=0$. Furthermore, in case of multiple integration of a function $f(t)$, the integration operational matrix of generalized block pulse series shows more accurate results than the conventional block pulse operational matrix $P$ and $\bar{P}[8]$.

$$
\bar{P}_{g}=\left(\frac{T}{m}\right)^{k+1} \frac{1}{(k+2) !}\left[\begin{array}{ccccc}
f_{1} & f_{2} & f_{3} & \ldots & f_{m} \\
0 & f_{1} & f_{2} & \ldots & f_{m-1} \\
0 & 0 & f_{1} & \ldots & f_{m-2} \\
: & : & : & \ldots & \vdots \\
0 & 0 & 0 & \ldots & f_{1}
\end{array}\right]
$$

The generalized block pulse function $\emptyset(t)$ is shown in Figure 5 for $i=0,1,2, \ldots, m-1$.

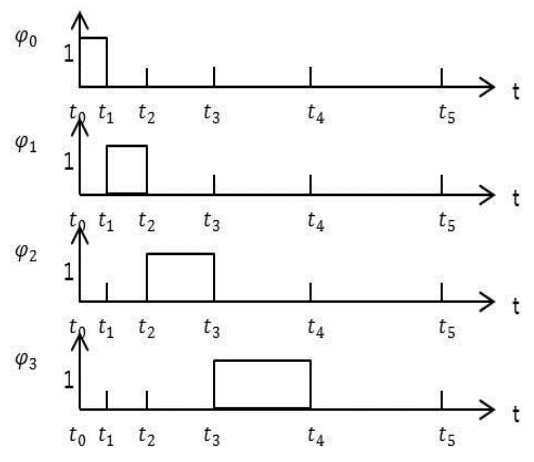

Figure 5. Generalized Block Pulse Function with $M=4$ 
Now we can express the $\mathrm{k}$ times integral of a function $f(t)$ using the generalized integration operational matrix $\bar{P}_{g}$ and its functions [9].

$$
\int_{0}^{t} \ldots \int_{0}^{t} f(t) d t \ldots d t \cong F^{T} \bar{P}_{g}^{k} \emptyset(t)
$$

The computation of using $\bar{P}_{g}^{k}$ is much smaller than that of using $P^{k}$ and $\bar{P}^{k}$ especially for case of large numbers $m$ and $\mathrm{k}$. The calculative defects or errors are also reduced by using the generalized integration operational matrix.

\section{Reduction Algorithm using Generalized Block Pulse Transform}

Let's consider a linear system which is described by a transfer function to express the output $y(t)$ using the generalized block pulse function.

$$
G(s)=\frac{Y(s)}{U(s)}=\frac{a_{1} s^{m-1}+a_{2} s^{m-2}+\cdots+a_{m-1} s+a_{m}}{s^{n}+b_{1} s^{n-1}+\cdots+b_{n-1} s+b_{n}}
$$

In equation (3.1), $Y(s)$ and $U(s)$ is the Laplace transform of $y(t)$ and $u(t)$ respectively with all initial conditions as zero. And we can get simple expression of equation (3.1) as

$$
\begin{aligned}
& Y_{i}(s)=s^{-1} Y(s)_{i-1} \\
& U_{i}(s)=s^{-1} U(s)_{i-1}
\end{aligned}
$$

The output $y(t)$ and input $u(t)$ of high order system can be expressed using the generalized block pulse series.

$$
\begin{aligned}
y(\mathrm{t}) & =\sum_{i=1}^{m} Y_{i} \varnothing_{i}(t)=Y_{h}^{T} \emptyset(t) \\
& u(\mathrm{t})=\sum_{i=1}^{m} U_{i} \varnothing_{i}(t)=U_{h}^{T} \emptyset(t)
\end{aligned}
$$

And from equation (3.2) and (3.3), we can express the output $y_{l}(t)$ and input $u_{l}(t)$ of lower order system using the generalized integration operational matrix.

$$
\begin{gathered}
y_{l}(\mathrm{t})=\sum_{i=1}^{n} Y_{i} \bar{P}_{g}^{i} \emptyset(t)=Y_{l}^{T} \bar{P}_{g}^{n} \emptyset(t) \\
u_{l}(\mathrm{t})=\sum_{i=1}^{n} U_{i} \bar{P}_{g}^{i} \emptyset(t)=U_{l}^{T} \bar{P}_{g}^{n} \emptyset(t)
\end{gathered}
$$

Where $T$ denotes transpose. The $Y_{l}^{T}$ is generalized block pulse function coefficient vector of the output and the $U_{l}^{T}$ is input coefficient vector of lower order system. And we use the substitution $\tau=\frac{t}{T}$, then we can get the coefficient $Y_{l}^{T}$ and determine lower order system output $y_{l}(t)$ using equation (3.6) and (3.7).

$$
Y_{l}^{T}=\left[I+\sum_{i=1}^{n}\left(b_{i} T_{i}\right) \bar{P}_{g}^{n}\right]^{-1}\left[\sum_{i=1}^{n}\left(a_{i} T_{i}\right) U_{l}^{T} \bar{P}_{g}^{n}\right]
$$

Now we can express the transfer function of reduced model with $p$ inputs and $q$ outputs. In this case, the definition of a transfer function is easily extended to a multivariable system with multiple inputs and outputs. So we can express lower order MIMO transfer function $\breve{G}_{M}(s)$ in matrix form.

$$
\begin{gathered}
\breve{G}_{M}(s)=\frac{\breve{Y}(s)}{\breve{U}(s)}=\frac{\breve{a}_{1} s^{r-1}+\breve{a}_{2} s^{r-2}+\cdots+\breve{a}_{f-1} s+\breve{a}_{f}}{\breve{b}_{1} s^{l-1}+\breve{b}_{2} s^{l-1}+\cdots+\breve{b}_{h-1} s+\breve{b}_{h}} \\
\breve{G}_{M}(s)=\left[\begin{array}{cccc}
L_{11}(s) & L_{12}(s) & \cdots & L_{1 p}(s) \\
L_{21}(s) & L_{22}(s) & \cdots & L_{2 p}(s) \\
\vdots & \vdots & \cdots & \vdots \\
L_{q 1}(s) & L_{q 2}(s) & \cdots & L_{q p}(s)
\end{array}\right]
\end{gathered}
$$

Where $m>r$ and $n>l$. In general, if a reduced MIMO system has $p$ inputs and $q$ outputs, the transfer function between $j$ th input and $i$ th output is defined as 


$$
L_{i j}(\mathrm{~s})=\frac{\breve{Y}_{i}(s)}{\breve{U}_{j}(s)}
$$

Where $\mathrm{i}=1,2, \ldots q, j=1,2, \ldots, p$

Now we can apply equation (3.6), (3.7) and (3.8) to determine any element of MIMO transfer function of lower order system. It can be obtained by

$$
L_{i j}(\mathrm{~s})=\left[I+\sum_{i=1}^{n}\left(b_{i} T_{i}\right) \bar{P}_{g}^{n}\right]^{-1}\left[\sum_{i=1}^{n}\left(a_{i} T_{i}\right) U_{l}^{T} \bar{P}_{g}^{n}\right]\left[U_{l}^{T}\right]^{-1}
$$

Therefore we can define the $L_{i j}$ (s) of reduced transfer function of MIMO model using the generalized integration operational matrix.

\section{Simulations}

\subsection{Example 1}

Let's consider and apply the suggested algorithm to a high order MIMO system with three inputs and outputs as [10]

$$
G(s)=\left[\begin{array}{lll}
G_{11} & G_{12} & G_{13} \\
G_{21} & G_{22} & G_{23} \\
G_{31} & G_{32} & G_{33}
\end{array}\right]
$$

The $G(s)$ of this example has $3 \times 3$ matrices. For convenience, we have just consider two elements of the high order MIMO transfer function $G(s)$.

$$
\begin{aligned}
G_{11}(s) & =\frac{1}{0.122 s^{3}+0.831 s^{2}+1.43 s+1} \\
G_{33}(s) & =\frac{1}{0.04 s^{3}+0.58 s^{2}+1.07 s+1}
\end{aligned}
$$

As the result, we can obtain the reduced order MIMO transfer function $\breve{G}_{M}(s)$ by applying proposed algorithm based on generalized block pulse function and operational matrix as

$$
\breve{G}_{M}(s)=\left[\begin{array}{ccc}
L_{11}=\frac{1}{0.854 s+1} & L_{12} & L_{13} \\
L_{21} & L_{22} & L_{23} \\
L_{31} & L_{32} & L_{33}=\frac{1}{1.98 s+2.01}
\end{array}\right]
$$

In equation (4.3), the original third order system is reduced to first-order system as shown in $L_{11}$ and $L_{33}$. For

\subsection{Example 2}

Now let's convert the following third-order system transfer function to the reduced model using the presented method based on the generalized integration operational matrix. Table 1 is represents the coefficients of high order system.

$$
\frac{Y(s)}{U(s)}=\frac{s+4}{s^{3}+6 s^{2}+11 s+6}
$$


Table 1. High Order System

\begin{tabular}{|c|c|}
\hline $\begin{array}{c}\text { Paramet } \\
\text { ers }\end{array}$ & $\begin{array}{c}\text { coefficients of high order } \\
\text { system }\end{array}$ \\
\hline$h_{0}$ & 0.49119 \\
\hline$h_{1}$ & -0.15510 \\
\hline$h_{2}$ & -0.09327 \\
\hline$h_{3}$ & -0.07991 \\
\hline$h_{4}$ & -0.04149 \\
\hline$h_{5}$ & -0.03388 \\
\hline$h_{6}$ & -0.01187 \\
\hline$h_{7}$ & -0.00688 \\
\hline
\end{tabular}

For this, we have used the generalized block pulse function with $m=8$ and time scale factor $t_{f}=6$, then we can get reduced model parameters and second order transfer function as shown in equation (4.6) and Table 2. Table 2 represents the coefficients of lower order system obtained using the proposed algorithm.

$$
\frac{Y_{l}(s)}{U_{l}(s)}=\frac{\breve{a}_{1} s+\breve{a}_{2}}{s^{2}+\breve{b}_{1} s+\breve{b}_{2}}
$$

Table 2. Lower Order System

\begin{tabular}{|c|c|}
\hline parameters & coefficients of lower order system \\
\hline$\breve{b}_{2}$ & 2.65783 \\
\hline$\breve{b}_{1}$ & 1.79107 \\
\hline$\breve{a}_{2}$ & -0.01707 \\
\hline$\breve{a}_{1}$ & 1.13465 \\
\hline
\end{tabular}

From the examples, the proposed algorithm based on generalized integration operational matrix is useful in determining the lower order MIMO system from an original high order model.

\section{Conclusions}

The approximation algorithm for MIMO transfer function based on the generalized block pulse integration operational matrix has been proposed. In control theory, if the original MIMO system is stable, we can obtain a reduced MIMO model by removing insignificant eigenvalues of the original MIMO system. But the reduced first order MIMO model which was reduced provides more inferior approximation to the original third order MIMO model than the second order system. Presented approximation algorithm is simple and useful for determining a reduced MIMO system. And the recursive processing of multiple integrals based on the generalized block pulse integration operational matrix is superior to the conventional method.

\section{Acknowledgements}

"This research was financially supported by the Ministry of Education (MOE) and National Research Foundation of Korea(NRF) through the Human Resource Training Project for Regional Innovation (No. 2014H1C1A1066414).”

\section{References}

[1] K. R. Palanisamy and V. P. Arunachalam, "Solution of variational problems using block pulse functions", Int. J. of Systems, vol. 16, no. 2, (1985), pp. 257-267.

[2] H. Hwang and Y. P. Shin, "On the operational matrices of block pulse functions", Int. J. of Systems, vol. 17, no.10, (1986), pp. 1489-1498. 
[3] J. H. Park, "Trajectory optimization for large scale systems via block pulse functions and transformations", Int. J. of CA, vol. 5, no. 4, (2012), pp. 39-48.

[4] J. H. Park and R. D. Oh, "Simplification of high order transfer function using general block pulse functions and transforms in frequency domain", Int. J. of Applied Engineering Research, vol. 9, no. 23, (2014), pp. 18231-18239.

[5] C. H. Wang and R. S. Marleau, "System identification via generalized block pulse operational matrices", Int. J. of Systems, vol. 16, no. 11, (1985), pp. 1425-1430.

[6] W. L. Chen and C. Y. Chung, "New integral operational matrix in block pulse series analysis", Int. J. of Systems, vol. 18, no. 3, (1987), pp. 403-408.

[7] J. H. Park, "Transfer Function Approximation using Rationalized Haar Transform in Frequency Domain”, Int. J. of CA, vol. 7, no. 4, (2014), pp. 247-258.

[8] W. Chi-Hsu, "Generalized block pulse operational matrices and their applications to operational calculus", Int. J. Control, vol. 36, no. 1, (1982), pp. 67-76.

[9] W. Chi-Hsu and R. S. Marleau, "Recursive computational algorithm for the generalized block pulse operational matrix", Int. J. Control, vol. 45, no. 1, (1987), pp. 195-201.

[10] J. H. Park, "Algorithm for multivariable system reduction using generalized block pulse function", $6^{\text {th }}$ International Workshop on Electrical and Electronics, Jeju Island, Korea, (2015) April 15-17.

\section{Author}

Joon-Hoon Park, Ph. D., since 1991, he is currently Professor of Dept. of Control and Instrumentations Eng. of KNUT in Korea. He is interested in optimal control theory, system design and its applications 
International Journal of Control and Automation Vol. 8, No. 6 (2015) 